\title{
Meteorological and climate change themes at the 2010 International Conference on Emerging Infectious Diseases
}

N Wilson (nick.wilson@otago.ac.nz) ${ }^{1}$, D Lush² M G Baker $^{1}$

1. Department of Public Health, University of Otago, Wellington, New Zealand

2. MAF Biosecurity, Ministry of Agriculture and Forestry (MAF), Wellington, New Zealand

Citation style for this article:

Wilson N, Lush D, Baker MG. Meteorological and climate change themes at the 2010 International Conference on Emerging Infectious Diseases. Euro Surveill.

2010;15(30): pii=19627. Available online: http://www.eurosurveillance.org/ViewArticle.aspx?Articleld=19627

Article published on 29 July 2010

This report outlines selected highlights of presentations that took place at the 2010 International Conference on Emerging Infectious Diseases (ICEID) [1], held between 11 and 14 July 2010 at the Hyatt Regency Atlanta in Atlanta, Georgia, United States (US). The conference was organised by five national and international bodies and was attended by over 1,600 participants from a wide range of professional backgrounds. Abstracts for the posters and slide sessions have been published [2].

This year's conference marked its seventh occurrence and it had a number of prominent themes with the strongest being the $A\left(\mathrm{H}_{1} \mathrm{~N}_{1}\right)$ influenza pandemic that emerged in 2009. Another theme was around the drivers for the emergence of infectious diseases including human demographics (e.g. growth of megacities), international movements of people (of travellers and refugees), trade in animals and the smuggling of wildlife, and various other aspects of globalisation. Meteorological and climate change drivers, of growing international and European-focused interest [3,4], were also well covered at this conference. Considering this growing interest, we outlined the latter drivers in this brief conference report.

\section{Mosquito-borne diseases}

Wilson (Session $\mathrm{G}_{1}$ ) discussed the issue of warmer cities favouring mosquito breeding. This was referred to along with higher temperatures shortening extrinsic incubation periods, e.g. in the urban vector Aedes aegypti. Nevertheless, although climate change may be influencing diseases such as dengue virus infection, other human-driven activities may be dominant drivers of risk.

Various speakers mentioned the problem of dengue fever with its expanded global distribution and rising case burden, including increased risk for travellers (e.g. Barnett et al., Poster 418). Although the pattern of dengue outbreaks in China has been variable over the past two decades, researchers suggested that an 'increasing threat of imported dengue will continuously increase the risk of dengue epidemics in China' (Xu et al., Poster 60). Blackmore (Session 01) gave a presentation on an outbreak of locally transmitted dengue virus infection in the city of Key West Florida in 2009, the first such local transmission in 70 years. Blackmore et al. concluded that in terms of dengue fever, this was "the outbreak with the largest disease incidence identified in the continental United States in 60 years'. Subsequent to the initial 2009 outbreak, the occurrence of recent cases during 2010 may suggest that dengue virus continues to be present in this area. In addition to this outbreak, it was reported that there has been an increase in imported cases in Florida in the last decade (Blackmore, Session 01).

Work on West Nile virus (WNV) was reported in the Northern Great Plains region of the US where this virus invaded in 2002. Since then, the incidence of human cases has remained relatively high (Chuang et al., Poster 259). Data on mosquitoes and meteorological factors indicated that higher mosquito populations in a given month were associated with higher temperature and precipitation in the preceding month. Similarly, a study in British Colombia, Canada examined the emergence of WNV in 2009 (after a spread westward across the continent) (Roth et al., Poster 61). This work suggested that 'above average temperatures, low snow pack and resulting reduced stream flows may have caused the observed increase in Culex tarsalis numbers in 2009 that facilitated viral amplification and spillover into human and equine populations'.

Other mosquito-borne diseases described at the conference included the following

- Murphree presented on La Crosse encephalitis which appears to have emerged in East Tennessee, US (Session 01), but this pattern may reflect changes in surveillance as opposed to climatic or other factors.

- The re-emergence of chikungunya virus was referred to by Lieberman and Prince (i.e. in the Indian Ocean islands in 2005 , spread to the Indian subcontinent 
in 2006 and it caused a localised outbreak in Italy in 2007) (Poster 241). This work presented results of newly developed immunofluorescence assays for detecting the virus.

- Pogosta disease is a mosquito-transmitted viral infection caused by Sindbis virus. Work from Finland (Jalava et al., Poster 192) reported that rainfall was a predictor for the occurrence of this disease in humans (with time lags of one, two and three months) and with the rainfall probably influencing the risk of mosquitoes transmitting the disease. However, the data for Finland show no long term increasing trend and the disease appears to have a seven-year cycle which may be related to a role of tetraonid birds (grouse) [5].

The overall pattern of the studies presented on mosquito-borne diseases at the conference was of expanded ranges for disease incidence. Meteorological and climate change factors were identified for some of these patterns but clearly many other factors are involved and may be more important drivers.

\section{Tick-borne diseases}

One of the most impressive presentations at the conference was on tick-borne encephalitis (TBE) in central and eastern Europe by Randolph (Session D3). Very detailed work suggested an overall pattern of increased TBE associated with climate change (i.e. increased TBE associated with increased temperature in spring). However, many other factors were described as contributing to the heterogeneous patterns of TBE incidence and trends including changes in: vegetation cover (e.g. favouring deer); land tenure (impacting on human access to land); and human use of the land (e.g. for leisure activities and for collecting firewood and food from forests).

Data from Ukraine presented by Biletska et al. (Poster 506) indicated an increase in Lyme disease from 2000 to 2008. Discussion with the author indicated that warmer weather in Ukraine may be playing a role but that other factors are also plausible (e.g. increased human exposure in forested areas).

Various presentations described tick-borne diseases in the US and Kemperman (Session 01) noted the nationwide increase in Lyme disease and human anaplasmosis (previously known as human granulocytic ehrlichiosis) in recent years. An increase in Powassan virus disease in North America was also reported by McQuiston (Session 01) along with the emergence of Rocky Mountain spotted fever in Arizona. The risk for the latter was possibly linked to weather (the 'monsoon season' in Arizona). Others described the evidence for the spread of babesiosis to new states (Lutz et al., Poster 57; and Mosites et al., Poster 310). In a study from Minnesota, Kemperman presented evidence for an increase in the number of counties reporting cases of three tick-borne diseases: Lyme disease, human anaplasmosis, and babesiosis (Session 01). In a study by Rockwell et al. on an apparent increase in reporting of human monocytic ehrlichiosis, there were also data to suggest an expansion of the geographic range (northward) of the implicated 'Lone Star tick' (Poster 26). Nevertheless, the role of meteorological and climate factors in these various tick-borne disease studies in the US was not considered in detail by the presenters, and it is plausible that improvements in laboratory tests and in surveillance are playing a role for the apparent increase in these diseases.

\section{Food and water-borne diseases}

The threat that climate change poses to water supply quality (e.g. via droughts or flooding) was raised at the conference. A presentation by Chen (Session E3) dealt with an outbreak of leptospirosis associated with a typhoon-related flooding event in Taiwan. Another presentation by Nichols (Session D3) described the relationship between excess cumulative rainfall in the previous seven days and drinking water related outbreaks in the United Kingdom. Furthermore, this study concluded that low rainfall levels (for the three weeks before an outbreak) were significantly associated with increased outbreak risks.

Henao (Session 04) presented a study on foodborne disease trends in the US for the 1996 to 2009 period using FoodNet surveillance data which indicated a decline in salmonellosis. This would suggest that climate change is not a substantive driver for salmonellosis in the US setting, even though higher ambient temperatures appear to increase the risk according to the literature [6] and to other data presented at this conference (Wilson et al., Poster 59). By contrast, Vibrio infections increased substantially over this 1996 to 2009 period in the US (Henao, Session 04). This increase in Vibrio infections was seen for both FoodNet and the "Cholera and Other Vibrio Illness Surveillance System (COVIS)' in a presentation by Vugia (Session $\mathrm{M}_{4}$ ). While it was speculated that the increase in sea surface temperature may account for some of the increase in Vibrio infections, other factors that are likely to be contributing include trends in shellfish consumption patterns and recreational contact with contaminated water.

In a presentation on Naegleria fowleri and primary amoebic meningoencephalitis in the US, Yoder (Session J6) reported no clear trend in incidence since 1978. Nevertheless, given that the occurrence of this organism is associated with warmer water temperatures, it was noted that there was 'potential' for increased risk with climate change.

\section{Airborne diseases}

Soebiyanto and Kiang presented a study of forecasting of seasonal influenza using climatic data from satellites and ground-based meteorological stations (Poster 503). This work considered that the resulting model was able to identify the timing of influenza peak activity 'reasonably well'. A study from Brazil by Alonso et al. on influenza and respiratory syncytial virus found 
that both these infections in children were positively correlated to rainfall even after adjusting for seasonality (Poster 492). However, they were only negatively correlated to air temperature when seasonality was not controlled for in the model.

Garrison et al. reported in a review of legionellosis outbreaks globally that cooling towers were the environmental source for $41 \%$ of outbreaks and 'decorative pools or fountains' as the source of $5 \%$ of outbreaks (Poster 183). These findings are of note given that if the use of cooling towers and urban fountains become more prevalent in some countries as a result of climate change, then this may impact on legionellosis risk.

\section{Other infectious diseases}

A detailed historical study in Mexico by Acuña-Soto identified that typhus and haemorrhagic fevers of unknown aetiology were strongly associated with drought (Poster 186 and Session K1). Climatic factors were also suggested as contributing to the historical patterns of yellow fever, influenza and cholera in Mexico.

Other work of note included:

- a study on Q fever in the Netherlands by van der Hoek et al., which reported that low ground water levels were associated with increased risk and wet soil with decreased risk (Poster 200);

- a study on Crimean-Congo haemorrhagic fever in Pakistan from 1991 to 2007 by Tariq which reported an association with drought among a range of other risk factors (Poster 51);

- a review of a decade of emerging and re-emerging outbreaks of arboviral disease in livestock in Israel by Brenner which raised the potential role of climate change as a contributing factor (Poster 326);

- a review by Wilson et al. on climate change and infectious diseases in New Zealand which reported the results of modelling work and evidence for a temperature gradient for risk of salmonellosis (Poster 59);

- a review of climate change and wildlife health by Sleeman et al., who reported that there was convincing evidence for climate change impacts on animal life cycles and on the geographic range and distribution of diseases and disease vectors (Poster 258). These authors also described a research agenda around studying this topic further.

\section{Discussion and conclusions}

This conference included a wide range of data of relevance to considering meteorological and climate change related impacts on infectious diseases. Some of the studies presented have recently been published in the journal literature, but many of the studies appear to have been presented for the first time at this conference. The overall impression was one of concern around how climate change may be currently impacting on various diseases (particularly vector-borne diseases), and how any such effects may increase in the future.
Nevertheless, for many of these diseases, other risk factors relating to human activities may be more important and uncertainties are often large, especially for rarer diseases where there have been improvements in diagnostic tests and surveillance methods. A form of 'publication bias' may also play a role in determining which presentations and posters get offered and selected for such conferences.

Given the growing international concern around climate change, including in a recent US National Research Council Report [7], there is a need for more intensive climate change mitigation and adaptation strategies globally. In the infectious diseases area, a key adaptation strategy will be improved control of vector-borne diseases. But improvements in surveillance and research on whether and how various infectious diseases are influenced by meteorological patterns and climate change are also needed, especially research that considers interactions with other risk factors. Conference organisers can also play a role in designing session themes that allow this topic area to be explored in a coherent way and with panel sessions that attempt to integrate overall pictures of emerging evidence and appropriate research agendas.

\section{References}

1. 2010 International Conference on Emerging Infectious Diseases (ICEID). Atlanta: ICEID Conference Organizers; 2010. Available from: http://www.iceid.org/.

2. 2010 International Conference on Emerging Infectious Diseases: Program and abstracts book. Atlanta: ICEID Conference Organizers; 2010.

3. Semenza JC, Menne B. Climate change and infectious diseases in Europe. Lancet Infect Dis. 2009;9(6):365-75.

4. European Centre for Disease Prevention and Control (ECDC). Meeting Report: First meeting of ECDC Expert Group on Climate Change. Stockholm: ECDC; 2009. Available from: http://www. ecdc.europa.eu/en/publications/Publications/o910_MER First_Meeting_of_ECDC_Expert_Group_on_Climate_Change. pdf

5. Sane J, Guedes S, Kurkela S, Lyytikainen O, Vapalahti O. Epidemiological analysis of mosquito-borne Pogosta disease in Finland, 2009. Euro Surveill. 2010;15(2):pii=19462. Available from: http://www.eurosurveillance.org/ViewArticle. aspx?Articleld $=19462$

6. Kovats RS, Edwards SJ, Hajat S, Armstrong BG, Ebi KL, Menne B. The effect of temperature on food poisoning: a time-series analysis of salmonellosis in ten European countries. Epidemiol Infect. 2004;132(3):443-53.

7. Committee on Stabilization Targets for Atmospheric Greenhouse Gas Concentrations; National Research Council. Climate stabilization targets: Emissions, concentrations, and impacts over decades to millennia. Washington, DC: National Academies Press; 2010. Available from: http://www.nap.edu/ openbook.php?record_id=12877\&page $=\mathrm{R} 1$. 